\title{
First-Order Statistics Prediction for a Propagation Channel of Arbitrary Non-Geostationary Satellite Orbits
}

\author{
Milan KVICERA, Pavel PECHAC \\ Faculty of Electrical Engineering, Czech Technical University in Prague, Technicka 2, 16627 Prague, Czech Republic \\ kvicemil@fel.cvut.cz, pechac@fel.cvut.cz
}

\begin{abstract}
A method enabling the prediction of the firstorder statistics of received signal levels for arbitrary nongeostationary satellite orbits based on a reference dataset for a wide range of elevation angles is introduced for azimuth-independent scenarios and high elevation angles. The method is further validated by experimental data obtained during measurements with a remote-controlled airship utilized as a pseudo-satellite. These experimental trials were performed at a frequency of $2.0 \mathrm{GHz}$ at two scenarios at Stromovka Park in Prague, Czech Republic, in August 2013 and March 2014. An excellent match between the predicted and actual cumulative distribution functions of received signal levels was identified for both scenarios.
\end{abstract}

\section{Keywords}

Satellite-to-Earth propagation, channel measurements, modeling, vegetation

\section{Introduction}

Central to the design of a satellite system are the constellation and selected orbit which determine the elevation and azimuth angles at which a user shall receive a direct signal. The elevation and azimuth angle toward a particular satellite can remain the same for a fixed receiver, as in case of a geostationary Earth orbit, or change dramatically when considering for example a highly elliptical orbit. This has a strong influence on the corresponding satellite-to-Earth propagation channel as, based on user surroundings, the signal may be shadowed differently determined by the direction of the incoming signal. To predict such behavior, a number of various propagation channel models for different scenarios can be found in the literature, see for example [1-8]. Generally, such models need to be based on available experimental data; however, obtaining suitable experimental data is demanding. It is common to utilize a so-called pseudo-satellite which may be in a form of a transmitter (Tx) placed on a helicopter [9-11] or a remote-controlled airship [7], [12], [13], at a crane or the upper-most point in the surroundings for fixed-elevation measurements [14-16], or even collect data from an existing satellite [17-19].
However, it is not feasible to obtain experimental data for all the combinations of azimuth and elevation angles observed by a user on Earth when considering a particular non-geostationary satellite system. Instead, respecting high costs of experimental campaigns when using a pseudosatellite, pre-defined flight paths, such as a star-pattern [7], circle [11], or hemisphere [10], are chosen for selected scenarios.

Considering azimuth-independent scenarios identified in the text as regular, such as a densely vegetated area, only one set of reference experimental data in a vast range of elevation angles at an arbitrary azimuth should be sufficient to predict received signal characteristics for arbitrary non-geostationary satellite orbits leading to less demanding experimental campaigns. Such a novel approach would follow [20], where a probability density function (PDF) of elevation angles between a user and a low Earth orbit satellite is utilized to obtain resulting rain attenuation time series. However, such an experiment has not been documented in the literature and needs to be validated. Thus, a series of measurements at $2.0 \mathrm{GHz}$ at Stromovka Park in Prague, Czech Republic, were carried out in 2013 and 2014. Throughout these trials, a remote-controlled airship was utilized as a pseudo-satellite following pre-defined flight paths according to sub-satellite points of selected Galileo and Iridium satellites. Both the left- (LHCP) and right-handed (RHCP) circularly polarized signals were transmitted from the airship towards a receiver located at two different scenarios. Unlike [21], where four different distributions were fitted to the first-order statistics of experimental data obtained previously at Stromovka Park with a low sampling rate of $100 \mathrm{~Hz}$, this paper presents a method how to obtain a cumulative distribution function (CDF) of received signal levels for various non-geostationary satellite orbits based on a reference dataset for the case of azimuth-independent scenarios. Details of the experimental campaign are provided in Sec. 2, the data processing method is described in Sec. 3, while the results and discussion are given in Sec. 4.

\section{Measurement Setup and Trials}

The measurement setup used during the trials was as follows. A remote-controlled airship carried a Tx, the same 
type as in [7], connected to an LHCP and an RHCP planar wideband antenna attached to a positioner enabling an instant pointing towards the receiver (Rx) location based on the airship GPS coordinates. Unmodulated continuous wave signals with a fixed output power of $27 \mathrm{dBm}$ were transmitted at frequencies of $2.00106 \mathrm{GHz}$ and $2.00086 \mathrm{GHz}$ by the LHCP and RHCP antenna, respectively. Unlike [7] and [12], to obtain the received signal levels of both the co-polarized and cross-polarized components of the transmitted signals, a dual-polarized rectangular patch antenna was connected by an H-hybrid and two power splitters to a sensitive, custom-made, four-channel receiver with a low noise floor of $-126 \mathrm{dBm}$ for a measurement bandwidth of $12.5 \mathrm{kHz}$. The receiver provided a $10-\mathrm{kHz}$ sampling rate and its first two channels were tuned to $2.00106 \mathrm{GHz}$ and the remaining two were tuned to $2.00086 \mathrm{GHz}$. The height of the upwards-pointing receiving antenna was 1.5 meters and the altitude of the airship was kept approximately 200 meters above ground level at a near-constant speed of $8 \mathrm{~m} / \mathrm{s}$. Similar to [7], recorded signal levels were recalculated to a uniform distance of $20 \mathrm{~km}$ to eliminate the influence of free space loss for different distances between the Tx and Rx. Further, data obtained during periods of airship pitch and roll of more than 15 degrees were removed as they represent gusty conditions during which the Tx antenna positioner did not perfectly keep the direction towards Rx.

The airship followed pre-defined flight paths over the vegetated area of Stromovka Park according to the selected typical sub-satellite points of the Galileo PFM and Iridium 98 satellite for the location of Prague $\left(50.08^{\circ} \mathrm{N}, 14.43^{\circ} \mathrm{E}\right)$, see Fig. 1. Two scenarios, marked as A and B in Fig. 1 and shown in more detail in Fig. 2, were selected to represent regular scenarios independent of azimuth: a receiver located inside coniferous trees and within a group of tall deciduous trees, respectively. It should be noted that all the flight paths in Fig. 1 refer to scenario A and were thus slightly shifted for the case of scenario B. By performing the measurements in July 2013 and March 2014, represen-

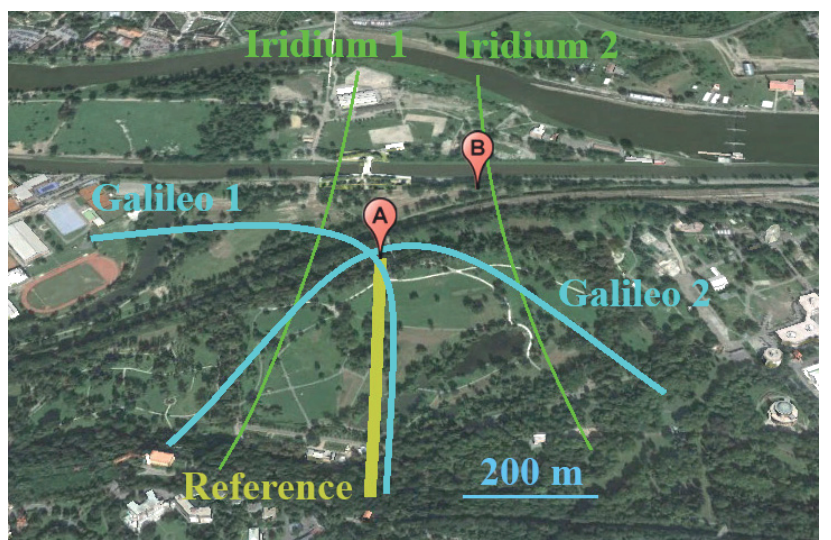

Fig. 1. Regular scenarios A and B at Stromovka Park together with the pre-defined airship flyovers simulating the Iridium 98 satellite (two almost north-south thin lines), Galileo PFM satellite (two wider curved lines) and the reference north-south flyover (widest line). (Image from Google Earth).
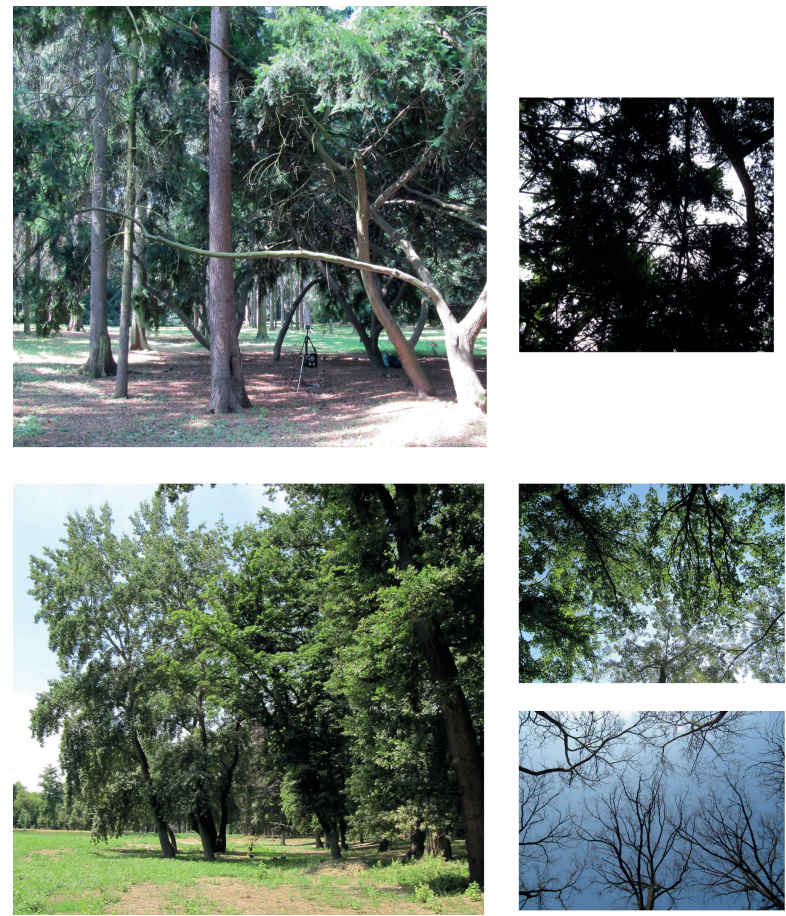

Fig. 2. Scenarios A (top left) and B (bottom left) in detail during the summer season together with the upwards views from the $\mathrm{Rx}$ during the summer and winter season (top right and bottom right). As scenario $\mathrm{A}$ is evergreen, only one upwards view is shown for both seasons.

tative experimental data were obtained for the actual satellite passes during both the summer and winter season. As scenario A is evergreen, it demonstrates the repeatability of the measurements while defoliation of scenario B represents different propagation conditions. Thus only these two scenarios can be utilized to obtain a statistically significant amount of experimental data and validate the method presented below in Sec. 3 .

It should be noted that although the utilized frequencies are at the upper part of L-band, higher than the actual Galileo or Iridium frequencies, the method introduced in the next section is limited by the azimuthal symmetry of a particular scenario rather than by a selected frequency. In addition, even though the airship simulated actual nongeostationary satellite systems, arbitrary flyovers could have been selected.

\section{Data Processing}

The experimental data were processed in the following way. The measurements were aimed at high elevation angles and, thus, the lowest elevation considered was 30 degrees from the Rx point of view. On the other hand, to avoid an insufficient amount of data for high elevation angles, the maximum considered elevation was 80 degrees. For the analysis described below, only the co-polarized components of the received LHCP and RHCP signals were considered. 
As a subsequent step, the entire range of elevation angles achieved during the north-south reference flyover (shown by the thick line in Fig. 2) was divided into fivedegree intervals and a PDF $p d f_{\text {reference, } i}(r)$ of received signal levels $r$ in $\mathrm{dB}$ was calculated by means of histogram for each elevation interval $i$. In contrast to [20], where segments of constant time are utilized, our approach requires constant elevation intervals as the simulated flyovers do not follow the actual time duration of the particular satellite pass. The choice of the interval size respected a statistically significant number of data samples (about 80000 and 40000 for the lowest and highest elevation interval, respectively) and, in addition, propagation conditions and antenna patterns are considered as not significantly varying within such an interval, similar to [7].

Consequently, for the Galileo or Iridium flyovers shown in Fig. 1, a PDF of the elevation intervals $p d f_{\Theta}(\theta)$ achieved during a particular flyover was calculated and thus the probability $p_{\Theta, i}(\theta)$ of each elevation interval $i$ was obtained. Then, following (1), a PDF of signal levels received during the reference flyover within an elevation interval $i, p d f_{\text {reference, } i}(r)$, was multiplied by $p_{\Theta, i}(\theta)$ so that a PDF of received signal level $p d f_{\text {predicted, } i}(r)$ was predicted for this elevation interval. After that, a PDF of received signal levels $p d f_{\text {predicted }}(r)$ for the whole range of elevation angles from 30 degrees up to 80 degrees was obtained according to (2), simply as a sum of the PDFs for every elevation interval.

$$
\begin{gathered}
p d f_{\text {predicted, } i}(r)=p_{\Theta, i}(\theta) \cdot p d f_{\text {reference, } i}(r) \text { for } i=1 \ldots n, \\
p d f_{\text {predicted }}(r)=\sum_{i=1}^{n} p d f_{\text {predicted }, i}(r)
\end{gathered}
$$

where $n$ is the total number of elevation intervals. Obtaining a corresponding CDF is then straightforward by using (3)

$$
\operatorname{Pr}\left\{r \leq r_{t h}\right\}=\int_{-\infty}^{r_{t h}} p d f_{\text {predicted }}(r) d r .
$$

\section{Results and Discussion}

To analyze and quantify the quality of the overall match of the predicted and actual received signal level CDF for a particular satellite flyover, we calculated the mean and standard deviation of their difference for the whole range of percentages with a step of 0.01 . Corresponding results are then given in Tab. 1 with the exception of Iridium flyover number 2 for scenario $B$ during the summer season as the experimental data are not available.

Based on this table it is evident that overall similar results were obtained for scenarios A and B during the summer and winter season. Absolute values of maximum and minimum mean of differences are below $5 \mathrm{~dB}$ and about $0 \mathrm{~dB}$, respectively. Together, with maximum standard deviations below $2.5 \mathrm{~dB}$, these values indicate an excellent match of the predicted and actual CDFs. Such results also reflect the fact that even scenario $\mathrm{B}$, consisting of only five

\begin{tabular}{|c|c|c|c|c|c|}
\hline \multicolumn{2}{|c|}{} & \multicolumn{4}{|c|}{ Mean \pm standard deviation (dB) } \\
\hline \multicolumn{2}{|c|}{ Scenario } & Galileo 1 & Galileo 2 & Iridium 1 & Iridium 2 \\
\hline \multirow{2}{*}{$\begin{array}{c}\text { A, } \\
\text { sum. }\end{array}$} & LHCP & $-1.4 \pm 1.6$ & $0.8 \pm 1.5$ & $1.1 \pm 2.4$ & $-2.5 \pm 0.8$ \\
\cline { 2 - 6 } & RHCP & $-1.8 \pm 1.3$ & $1.2 \pm 0.8$ & $-0.2 \pm 2.1$ & $-2.1 \pm 0.7$ \\
\hline \multirow{2}{*}{$\begin{array}{c}\text { A, } \\
\text { winter }\end{array}$} & LHCP & $3.6 \pm 1.2$ & $1.9 \pm 1.4$ & $3.0 \pm 1.7$ & $0.0 \pm 0.8$ \\
\cline { 2 - 6 } & RCHP & $2.4 \pm 0.9$ & $1.2 \pm 1.4$ & $0.9 \pm 2.2$ & $1.2 \pm 1.1$ \\
\hline \multirow{2}{*}{$\begin{array}{c}\text { B, } \\
\text { sum. }\end{array}$} & LHCP & $-2.9 \pm 0.8$ & $-0.6 \pm 1.8$ & $0.8 \pm 2.1$ & Not avail. \\
\cline { 2 - 6 } & RHCP & $-4.9 \pm 0.9$ & $-3.1 \pm 2.0$ & $-3.8 \pm 1.3$ & Not avail. \\
\hline \multirow{2}{*}{$\begin{array}{c}\text { B } \\
\text { winter }\end{array}$} & LHCP & $-2.3 \pm 1.0$ & $-2.9 \pm 2.7$ & $-1.4 \pm 1.5$ & $-2.3 \pm 2.2$ \\
\cline { 2 - 6 } & RHCP & $-3.6 \pm 1.0$ & $-1.7 \pm 2.1$ & $-2.7 \pm 1.3$ & $0.8 \pm 1.7$ \\
\hline
\end{tabular}

Tab. 1. Mean and standard deviation of difference between predicted and measured CDF.

deciduous high-rose trees, with non-uniformly distributed sparse trunks and branches without leaves in the winter season, can still be considered as regular, although it could be assumed to be more dependent on azimuth than scenario A.

It should be noted that a certain level of inaccuracy is introduced into the predicted results due to the Rx antenna pattern not being perfectly azimuth independent. However, respecting the Rx antenna design, the maximum difference of gain for both the LHCP and RHCP polarization can be identified for the lowest elevations as only about $2 \mathrm{~dB}$ for an elevation of 30 degrees.

To illustrate results from Tab. 1, Figs. 3 and 4 represent selected actual, predicted and reference signal level CDFs for the Galileo satellite flyover number 2, RHCP signal at scenario A and B during the summer season, respectively. For these cases, the mean value of the differences is $1.2 \mathrm{~dB}$ and $-3.1 \mathrm{~dB}$, whereas the standard deviation of the differences is $0.8 \mathrm{~dB}$ and $2.0 \mathrm{~dB}$, respectively. Based on Figs. 3 and 4, such values lead to a very good visual match of the predicted and actual signal level CDF, even for the worse case.

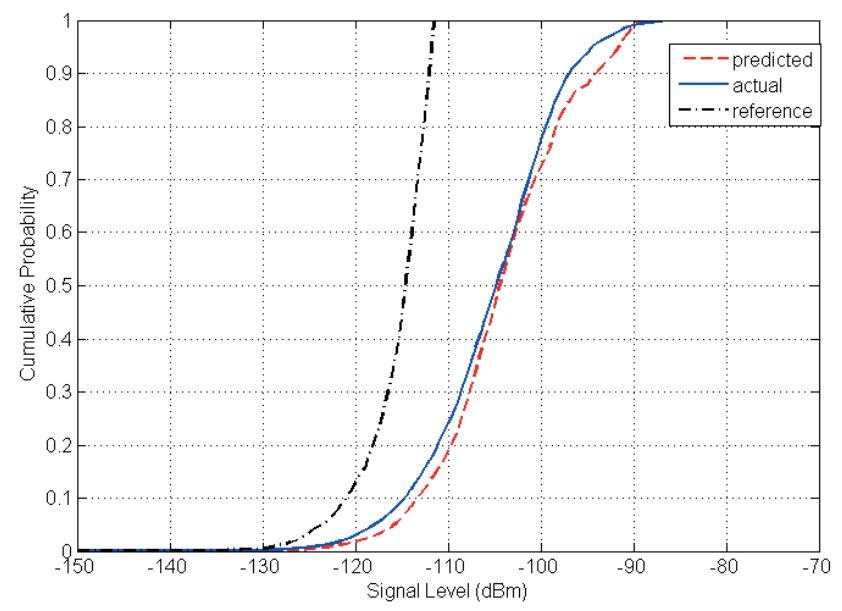

Fig. 3. An example of a match between the predicted and actual signal level CDF for the case of the RHCP signal during the Galileo satellite flyover number 2 at scenario A within the summer season. It corresponds to values of $(1.2 \pm 0.8) \mathrm{dB}$ taken from Tab. 1 . 


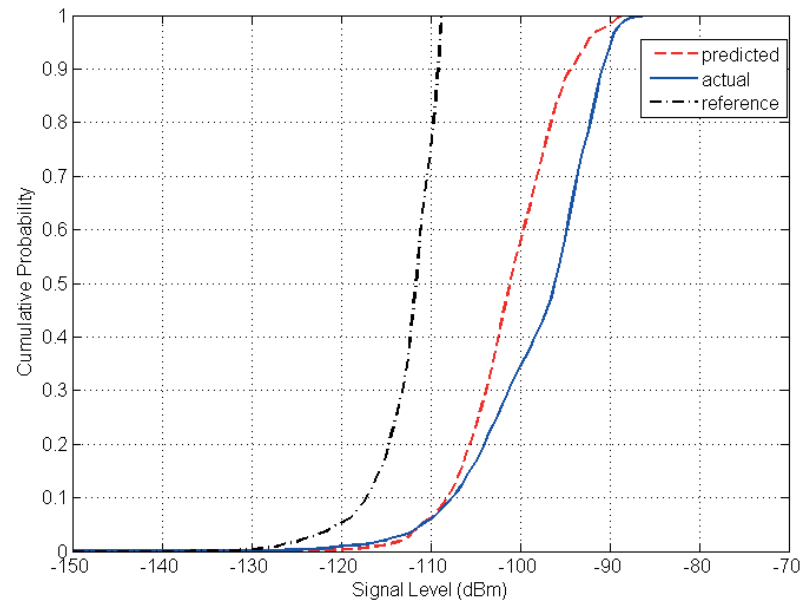

Fig. 4. An example of a match between the predicted and actual signal level CDF for the case of the RHCP signal during the Galileo satellite flyover number 2 at scenario B within the summer season. It corresponds to values of $(-3.1 \pm 2.0) \mathrm{dB}$ taken from Tab. 1 .

To summarize, based on Tab. 1, it can be stated that an overall very good match of the prediction was achieved for both scenarios $\mathrm{A}$ and $\mathrm{B}$ during summer and winter. Based on the large experimental dataset which has been processed, the presented prediction method can be considered as successfully validated for the case of high elevation angles and regular scenarios.

\section{Conclusion}

We have presented a method enabling the prediction of the first-order statistics of signal levels received at high elevation angles considering arbitrary satellite orbits and azimuth-independent scenarios. This method is based on utilizing a reference dataset for a wide range of elevation angles at one azimuth only. The validity of this method was demonstrated at a frequency of $2.0 \mathrm{GHz}$ on a significant number of experimental datasets obtained by using a remote-controlled airship simulating selected Galileo and Iridium satellite passes over two vegetated scenarios during both the summer and winter season. It was also shown that the proposed method can be utilized for common, not perfectly azimuth-independent scenarios, which would result in a significant decrease of complexity of corresponding experimental campaigns. Furthermore, as a consequence of the proposed method, if data obtained for the case of an existing satellite system are available, they may be utilized as a reference dataset to predict the first-order statistics of received signal levels at a similar frequency for a different constellation of the satellite system, at least as an initial estimation, without the considerable expense of a measurement campaign.

\section{Acknowledgements}

This publication was supported by the European social fund within the framework of realizing the project
„Support of inter-sectoral mobility and quality enhancement of research teams at the Czech Technical University in Prague", CZ.1.07/2.3.00/30.0034.

\section{References}

[1] JOST, T., CARRIE, G., PEREZ-FONTAN, F., WANG, W. FIEBIG, U.-C. A Deterministic satellite-to-indoor entry loss model. IEEE Transactions on Antennas and Propagation, 2013, vol. 61, no. 4, p. 2223-2230. DOI: 10.1109/TAP.2012.2232898

[2] CHEFFENA, M., PEREZ-FONTAN, F. Land mobile satellite channel simulator along roadside trees. IEEE Antennas and Wireless Propagation Letters, 2010, vol. 9, p. 748-751. DOI: 10.1109/LAWP.2010.2060465

[3] AIT-IGHIL, M., LEMORTON, J., PEREZ-FONTAN, F., LACOSTE, F., THEVENON, P., BOURGA, C., BOUSQUET, M. SCHUN - a hybrid land mobile satellite channel simulator enhanced for multipath modelling applied to satellite navigation systems. In Proceedings of the 7th European Conference on Antennas and Propagation. Gothenburg (Sweden), 2013, p. 692-696.

[4] CARRIE, G., PEREZ-FONTAN, F., LACOSTE, F., LEMORTON, J. A generative MIMO channel model: encompassing single satellite and satellite diversity cases. In Proceedings of the 6th European Conference on Antennas and Propagation. Prague (Czech Rep.), 2012, p. 2454-2458. DOI: 10.1109/EuCAP.2012.6206627

[5] JEANNIN, N., PEREZ-FONTAN, F., MAMETSA, H.-J., CASTANET, L. Physical-statistical model for the LMS channel at $\mathrm{Ku} / \mathrm{Ka}$ Band. In Proceedings of the 5th European Conference on Antennas and Propagation. Rome (Italy), 2011, p. 3571-3575.

[6] ABELE, A., PEREZ-FONTAN, F., BOUSQUET, M., VALTR, P., LEMORTON, J., LACOSTE, F., CORBEL, E. A new physicalstatistical model of the land mobile satellite propagation channel. In Proceedings of the 4th European Conference on Antennas and Propagation. Barcelona (Spain), 2010, p. 1-5.

[7] KVICERA, M., PECHAC, P. Building penetration loss for satellite services at L-, S- and C-band: measurement and modeling. IEEE Transactions on Antennas and Propagation, 2011, vol. 59, no. 84, p. 3013-3021. DOI: 10.1109/TAP.2011.2158963

[8] ARNDT, D., HEYN, T., HEUBERGER, A., PRIETO-CERDEIRA, R., EBERLEIN, E. State modeling of the land mobile satellite channel with angle diversity. In Proceedings of the 6th European Conference on Antennas and Propagation. Prague (Czech Rep.) 2012, p. 3130-3144. DOI: 10.1109/EuCAP.2012.6206141

[9] MOLNAR, B., FRIGYES, I., BODNAR, Z., HERCZKU, Z., KORMANYOS, Z., BERCES, J., PAPP, I., JUHASZ, L. Characterisation of the satellite-to-indoor channel based on narrow-band scalar measurements. In Proceedings of the $8^{\text {th }}$ IEEE International Symposium on Personal, Indoor and Mobile Radio Communications. Helsinki (Finland), 1997, p. 1014-1018.

[10] PEREZ-FONTAN, F., HOVINEN, V., SCHONHUBER, M., PRIETO-CERDEIRA, R., DELGADO-PENIN, J. A., TESCHL, F., KYROLAINEN, J., VALTR, P. Building entry loss and delay spread measurements on a simulated HAP-to-indoor link at Sband. EURASIP Journal on Wireless Communications and Networking, 2008, vol. 2008, no. 5, 6 p. DOI:10.1155/2008/427352

[11] TESCHL, F., PEREZ-FONTAN, F., SCHONHUBER, M., PRIETO-CERDEIRA, R., TESCHL, R. Attenuation of spruce, pine, and deciduous woodland at C-band. IEEE Antennas and Wireless Propagation Letters, 2012, vol. 11, p. 109-112. DOI: 10.1109/LAWP.2012.2184253

[12] HORAK, P., PECHAC, P. Excess loss for high elevation angle links shadowed by a single tree: measurements and modeling. 
IEEE Transactions on Antennas and Propagation, 2012, vol. 60, no.7, p. 3541-3545. DOI: 10.1109/TAP.2012.2196944

[13] STEINGASS, A., LEHNER, A. Measuring the navigation multipath channel - a statistical analysis. In Proceedings of the 17th International Technical Meeting of the Satellite Division of he Institute of Navigation (ION GNSS 2004). Long Beach (CA, USA), 2004, p. 1157-1164.

[14] LACOSTE, F., LEMORTON, J., CASADEBAiG, L., ROUSSEAU, F. Measurements of the land mobile and nomadic satellite channels at $2.2 \mathrm{GHz}$ and $3.8 \mathrm{GHz}$. In Proceedings of the 6th European Conference on Antennas and Propagation. Prague (Czech Rep.), 2012, p. 2422-2426. DOI: 10.1109/EuCAP.2012.6206356

[15] JOST, T., WANG, W., FIEBIG, U.-C., PEREZ-FONTAN, F. Comparison of L- and C-Band satellite-to-indoor broadband wave propagation for navigation applications. IEEE Transactions on Antennas and Propagation, 2011, vol. 59, no. 10, p. 3899-3909. DOI: 10.1109 /TAP.2011.2163753

[16] KING, P. R., STAVROU, S. Low elevation wideband land mobile satellite MIMO channel characteristics. IEEE Transactions on Wireless Communications, 2007, vol. 6, no. 70, p. 2712-2720. DOI: $10.1109 /$ TWC.2007.051018

[17] TESCHL, F., HOVINEN, V., PEREZ-FONTAN, F., SCHONHUBER, M., PRIETO-CERDEIRA, R. Narrow- and wideband land mobile satellite channel statistics for various environments at Ku-band. In Proceedings of the 6th European Conference on Antennas and Propagation. Prague (Czech Rep.), 2012, p. 2464-2468. DOI: 10.1109/EuCAP.2012.6206670

[18] JOST, T., WANG, W., SCHUBERT, F., ANTREICH F., FIEBIG, U.-C. Channel sounding using GNSS signals. In Proceedings of the 5th European Conference on Antennas and Propagation. Rome (Italy), 2011, p. 3724-3728.

[19] Veltsistas, P., KAlABOUKAS, G., KONITOPOUlOS, G., DRES, D., KATIMERTZOGLOU, E., CONSTANTINOU, P. Satellite-to-indoor building penetration loss for office environment at $11 \mathrm{GHz}$. IEEE Antennas and Wireless Propagation Letters, 2007, vol. 6, p. 96-99. DOI: 10.1109/LAWP.2007.893070

[20] ARAPOGlOU, P.-D. M., PANAgOpoulos, A. D. A tool for synthesizing rain attenuation time series in LEO Earth observation satellite downlinks at Ka band. In Proceedings of the 5th European Conference on Antennas and Propagation. Rome (Italy), 2011, p. $1467-1470$.

[21] KOUROGIORGAS, C. I., KVICERA, M., SKRAPARLIS, D., KORINEK, T., SAKARELlOS, V. K., PANAGOPOULOS, A. D., PECHAC, P. Modeling of first-order statistics of the MIMO dual polarized channel at $2 \mathrm{GHz}$ for land mobile satellite systems under tree shadowing. IEEE Transactions on Antennas and Propagation, 2014, vol. 62, no. 10, p. 5410-5415. DOI: 10.1109/TAP.2014.2346186

\section{About the Authors ...}

Milan KVICERA was born in 1983. He received the M.Sc. degree and the Ph.D. degree in Radio Electronics from the Czech Technical University in Prague, Czech Republic, in 2008 and 2012, respectively. He is currently working as a postdoc researcher in the Department of Electromagnetic Field, Czech Technical University in Prague. His research interests are focused on radiowave propagation and satellite communication.

Pavel PECHAC received the M.Sc. degree and the Ph.D. degree in Radio Electronics from the Czech Technical University in Prague, Czech Republic, in 1993 and 1999 respectively. He is currently a Professor in the Department of Electromagnetic Field, Czech Technical University in Prague. His research interests are in the field of radiowave propagation and wireless systems. 